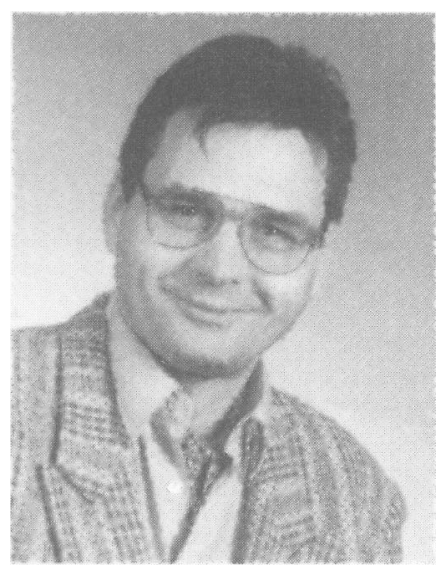

Prof. Dr. habil. Uwe Fichtner

die Schriftleitung einer Zeitschrift nachgerade prädestinierenden) Germanistik studierte er Geographie an der Universität Freiburg, legte hier 1975 das Erste Staatsexamen ab und bereitete sich anschließend auf die Promotion vor (Abschluß 1980), wobei die wissenschaftliche Kartographie zu einem seiner Schwerpunkte wurde. Anfangs als Tutor, später als Wissenschaftlicher Assistent, führte er über mehrere Jahre Übungen zur Entwurfslehre, Herstellung und Interpretation Topogra- phischer und Thematischer Karten durch; schließlich war er nach der Habilitation 1988 als Dozent für die Ausbildung im Fach Kartographie an der Universität Freiburg i. Br. allein verantwortlich. Während dieser Zeit befaßte er sich mit ersten Entwicklungen in der rechnergestützten Kartographie, die damals noch allein an der Großrechenanlage der Universität möglich war.

Seit 1988 gehört Dr. Uwe Fichtner zu den aktiven Mitgliedern des DGfK-Arbeitskreises Kartennutzung. Beruflich hat er seit 1993 eine Professur für Kartographie an der Fachhochschule Anhalt, Standort Bernburg, inne. Im Unterschied zum Standort Dessau, wo der Studiengang Vermessung der FH Anhalt beheimatet ist, werden in Bernburg Ingenieure der Fachrichtung Landespflege ausgebildet, für die Kenntnisse der Kartographie unabdingbar sind. Prof. Fichtner bietet jedes Semester eine vierstündige Vorlesung als Einführung in die Kartographie und GIS an, die durch Übungen vertieft wird. Außerdem ist er für die Ausbildung in Fernerkundung verantwortlich und führt entsprechende Projekte mit planungsbezogener Thematik durch.
Hierfür und für die Einführungsübungen stehen zwei ARC/INFOPools und ein ERDAS-Pool sowie die üblichen DTP-, DTM- und CAD-Programmpakete zur Verfügung.

Ich freue mich auf die Zusammenarbeit mit dem Kollegen Fichtner und bitte alle Leser der $\mathrm{KN}$, unter ihnen insbesondere die Landes- und Ortsvereine sowie die Arbeitskreise der DGfK, SGK und OeKK, ihn im Interesse unserer Zeitschrift bei seiner Arbeit zu unterstützen.

Die Qualität und Aktualität einer Fachzeitschrift und hier speziell die des Rubrikenteils hängt nicht nur von der Schriftleitung ab, sondern auch vom Engagement und der Mitarbeit aller potentiellen Autoren unter den Lesern. Daher unsere nachdrückliche Bitte: Geben Sie uns Hinweise auf aktuelle Ereignisse und Entwicklungen, schicken Sie uns entsprechende Berichte und Aufsätze, tragen Sie durch Kritik und Diskussion dazu bei, die KN weiterhin lebendig $\mathrm{zu}$ gestalten. Unsere Anschriften und "Zuständigkeitsbereiche" finden Sie an gewohnter Stelle im Impressum (S. 40).

Jürgen Dodt, Bochum

\title{
Veranstaltungen 1997
}

16. - 22. 2. 9. Internationale Geodätische Woche in Obergurgl, Tirol

18. - 21. 2. GIS '97 in Wiesbaden, Rhein-Main-Hallen

19. - 21. 2. Seminar „GIS und Kartographie im multimedialen Umfeld“, AK Kartographie und Geo-Informationssysteme der DGfK, Institut für Kartographie, Universität Hannover (s. KN 4/96, S. 153)

25. - 28. 2. Workshop „Geo-Informationssysteme“, FH Karlsruhe, Fachbereich Geoinformationswesen

16. - 18. 4. 3rd Joint European Conference and Exhibition on Geographical Information in Wien, Austria Center

4. - 8. 5. 46. Deutscher Kartographentag in Coburg, Kongreßhaus Rosengarten; Leitthema: „Kartographische Informationssysteme für Kommunen“ (s. KN 4/96, S. 155/156)

13. - 16. 5. geotechnica 1997, Internationale Fachmesse und Kongreß für Geowissenschaften und Geotechnik in Köln, Messe- und Ausstellungszentrum (s. KN 1/97, S. 28)

4. - 7. 6. 6. Österreichischer Geodätentag 1997 in Villach, Kärnten

19. - 21. 6. International Conference on Mass Media Maps, Freie Universität Berlin, Fachrichtung Kartographie

23. - 27. 6. ICC 97. 18. Internationale Kartographische Konferenz der IKV in Stockholm, Stockholm International Fairs; Leitthema „Maps and mapping in the information society“ (s. KN 5/96, S. 198)

2. - 4. 7. AGIT '97. 9. Symposium für angewandte Geographische Informationsverarbeitung in Salzburg, Universität (s. KN 6/96. S. 245 f.)

6. - 10. 7. 17. Internationale Konferenz zur Geschichte der Kartographie in Lissabon

17. - 19. 9. 81. Geodätentag/Intergeo in Karlsruhe

22. - 26. 9. 46. Photogrammetrische Woche in Stuttgart, Institut für Photogrammetrie der Universität (s. KN $1 / 97$, S. 27 f.)

7. - 8. 10. 34. Sitzung der Arbeitsgruppe „Automation in der Kartographie“, Institut für Kartographie, Technische Universität Dresden 